\title{
ADAPTABILIDADE E ESTABILIDADE FENOTÍPICA EM SOJA AVALIADA PELO MÉTODO DE TOLER ( $\left.{ }^{1}\right)$
}

\author{
LIZZ KEZZY DE MORAIS ( $\left.{ }^{2}\right)$; MARA FERNANDES MOURA $\left({ }^{3}\right)$; ROLAND VENCOVSKY $\left({ }^{4}\right)$; \\ JOSÉ BALDIN PINHEIRO $\left({ }^{4}\right)$
}

\begin{abstract}
RESUMO
O objetivo deste trabalho foi avaliar 69 genótipos de soja com diferentes ciclos de maturação em diferentes ambientes nos Estados de Mato Grosso e Mato Grosso do Sul utilizando o método de regressão não-linear proposto por TOLER (1990). O método propõe enquadrar os genótipos em cinco grupos, conforme o padrão de resposta A, B, C, D e E. Verificou-se que nas estimativas de qualidade ambiental houve diferenças em seus valores, permitindo inferir sobre os piores e melhores ambientes, Pedra Preta foi detectado como ambiente de melhor qualidade, com a maior estimativa, o que contribui para o aumento da média geral de ambientes. Ao classificar os genótipos com base na produtividade e no padrão de resposta verificou-se que genótipos com padrão de resposta duplamente desejável (grupo A, padrão convexo), atingiram produtividade alta com destaque para a linhagem FMT-42313 de ciclo precoce. Os genótipos de padrão E (padrão côncavo) não atingiram patamares satisfatórios de produtividade média como MSOY-8550 e Crixás. A produtividade dos genótipos com padrão de resposta unissegmentado $(B, C$ e D) foi variável, sendo a maioria enquadrados no grupo $C$, com resposta linear simples não desviando da resposta média dos ambientes, destacando-se a cultivar Conquista, com produtividade média e pouca variabilidade quando cultivada em ambientes distintos. O método proporciona vantagens estatísticas para a pesquisa sobre adaptabilidade e estabilidade de genótipos de soja, uma vez que discrimina esses materiais de base genética estreita em grupos de genótipos com diferentes padrões de resposta.
\end{abstract}

Palavras-chave: Glycine max, regressão não-linear, interação genótipo x ambiente, estabilidade, adaptabilidade.

\section{ABSTRACT \\ PHENOTIPIC STABILITY AND ADAPTABILITY IN SOYBEAN EVALUATED BY TOLER METHOD}

The objective of this work was to evaluate 69 soybean genotypes with different maturation cycles in different environments at Mato Grosso and Mato Grosso do Sul States in Brazil using the non-linear regression method of TOLER (1990). This methodology considers the fiting the genotypes in five groups, according to the response pattern A, B, C, D and E. It was observed that the environmental quality estimates had different values, allowing for inferences of worse and better environments. Pedra Preta was the best quality environment, having the greater estimative, that contributed to the increase of the general environment mean. The classification based on yield and on the response pattern showed that genotypes with double desirable response pattern (group A, convex pattern), presented high yield, mainly in the line early cycle FMT-42313. The pattern E genotypes (concave pattern) did not show satisfactory levels of mean yield as MSOY-8550 and Crixás. The response pattern of monosegmented genotypes ( $B, C$ and $D$ ) showed variable yield, being most of them clustered in group $C$, having a simple linear response that did not differ greatly from the mean response of the environments. Conquista cultivar had average yield and low variability in distinct environments. The methodology has statistic advantages for the analysis of adaptability and stability among soybean genotypes, since it discriminate genotypes with narrow genetic base in to groups with different response pattern.

Key words: Glycine max, non-linear regression, genotype x environment interaction, stability, adaptability

$\left({ }^{1}\right)$ Recebido para publicação em 21 de novembro de 2006 e aceito em 29 de setembro de 2007.

$\left({ }^{2}\right)$ Centro de Análise e Pesquisa Tecnológica do Agronegócio dos Grãos e Fibras, Instituto Agronômico-IAC, Caixa Postal 28, 13012-970 Campinas (SP). E-mail: lizz@iac.sp.gov.br (*) Autora correspondente.

$\left({ }^{3}\right)$ Centro de Análise e Pesquisa Tecnológica do Agronegócio das Frutas (IAC), Caixa Postal 11, 13200-970 Jundiaí (SP).

$\left({ }^{4}\right)$ Escola Superior de Agricultura "Luiz de Queiroz", Instituto de Genética, Universidade de São Paulo-USP, Caixa Postal 83, 13418-970, Piracicaba (SP). 


\section{INTRODUÇÃO}

Os programas de melhoramento de plantas visam à obtenção de genótipos com alta produtividade, estabilidade de produção e ampla adaptabilidade aos mais variados ambientes de cultivo. A interação de genótipos com os ambientes (GxA), definida como resposta diferencial dos genótipos à variação do ambiente, dificulta a seleção de genótipos amplamente adaptados. Além disso, essa interação pode inflacionar as estimativas de variância genética, resultando em superestimativa dos ganhos genéticos, esperados com a seleção, e com menor êxito, dos programas de melhoramento (DUARTE e VenCOVSKY, 1999).

A inconsistência na classificação dos genótipos, nos vários ambientes, é um dos principais desafios enfrentados pelos melhoristas. A ocorrência da interação pode ser detectada estatisticamente por uma usual análise conjunta de variância, de experimentos repetidos em mais de um ambiente, para a avaliação dos diferentes genótipos. Visando ao detalhamento do efeito da interação GxA pode-se realizar um estudo da estabilidade e adaptabilidade das cultivares. Esse procedimento analisa a variação ocorrida entre ambientes, para cada material genético, permitindo a seleção daqueles com tipo de resposta e padrão desejados.

Propostas metodológicas para se conhecer essa variação dos efeitos ambientais em cada genótipo, e a contribuição relativa de cada um para a interação total dos genótipos com ambientes foram primeiramente apresentados por Plaisted e Peterson (1959) e logo depois por WRIKE (1964). Essas metodologias deram informações sobre a interação GxA, mas não caracterizavam o comportamento individual dos genótipos sob várias condições ambientais. Posteriormente, novas metodologias foram apresentadas utilizando-se regressão linear, sendo o trabalho pioneiro nessa linha o de YATES e COCHRAN (1938), seguidos das metodologias descritas por FinLAY e WiLKINSON (1963) e EBERHART e RUSSEll (1966). Mais tarde, outros pesquisadores apresentaram refinamentos dessa técnica, entre os quais se podem citar Verma et al. (1978), Silva e BARRETo (1985) e Cruz et al. (1989).

Com o mesmo propósito de descrever as respostas genotípicas, TOLER (1990) apresentou um modelo de regressão não-linear que propõe aprimoramento em relação aos métodos que empregam regressão linear; procedimento que leva a classificar os genótipos em diferentes grupos, de acordo com seu padrão de resposta.

O método propõe distinguir entre uma reta linear e um padrão de resposta do genótipo (modelo unissegmentado) e uma combinação de duas retas com diferentes inclinações, sendo uma abaixo e outra acima da média ambiental (modelo bissegmentado), oferecendo como representação padrão de resposta convexo (duplamente desejável) e padrão de resposta côncavo (duplamente indesejável).

A literatura tem poucos trabalhos na interpretação de modelos não lineares quando comparados a modelos lineares para estudo da interação genótipo $x$ ambiente (GxA). Nesse contexto, o presente trabalho teve por objetivo avaliar o comportamento adaptativo de genótipos de soja pelo método de Toler nos Estados do Mato Grosso e Mato Grosso do Sul.

\section{MATERIAL E MÉTODOS}

Os experimentos foram desenvolvidos em dezembro do ano agrícola de 2002/2003, em sete locais, incluindo os Estados de Mato Grosso e Mato Grosso do Sul. Os locais com sua sigla de identificação foram: Rondonópolis (MT-111), Pedra Preta (MT-221), Sorriso (MT-528), Campo Novo do Parecis (MT-701), Costa Rica (MS-601), Chapadão do Sul (MS-621) e São Gabriel D'Oeste (MS-641). Foram avaliados genótipos de soja de três ciclos de maturação (precoce, médio e tardio), com número diferenciado de genótipos por ciclo. Os experimentos foram coordenados pela Fundação de Apoio à Pesquisa Agropecuária do Estado do Mato Grosso (Fundação MT). Foram avaliados 15 genótipos de ciclo precoce, 24 genótipos de ciclo médio e 30 genótipos de ciclo tardio.

Em cada local foram desenvolvidos três experimentos, cada um correspondendo a um dos ciclos de maturação. Utilizou-se o delineamento de blocos casualizados com três repetições. A parcela experimental foi representada por quatro fileiras de $5,0 \mathrm{~m}$ de comprimento, espaçadas de $0,5 \mathrm{~m}$ entre si. A parcela útil para avaliação do caráter produtividade de grãos foi de $4,0 \mathrm{~m}^{2}$, sendo assim avaliadas as duas fileiras centrais descartando-se $0,5 \mathrm{~m}$ de cada extremidade.

Utilizando-se os dados de produtividade de grãos, foram processadas as análises de variância de cada experimento (análises individuais). Em seguida, procedeu-se à análise conjunta de variância. Para essas análises, utilizou-se o procedimento GLM do aplicativo SAS (SAS INSTituTe, 2002). Para a análise conjunta, avaliou-se a presença de heterogeneidade entre as variâncias residuais, pelo teste $\mathrm{F}_{\text {máx }}$. Quando se detectou heterogeneidade, procedeu-se o ajuste dos graus de liberdade do erro médio e da interação, segundo o método de CoCRHAN (1954). Somente após esses ajustes foram feitas as interpretações quanto à 
significância do teste F. Detectada a presença de interação GxA significativa, foram realizadas posteriormente as análises de estabilidade e adaptabilidade, utilizando-se o método de Toler.

No procedimento de TOLER (1990), o índice ambiental é denotado $\mu_{\mathrm{j}}$, sendo, porém, estimado simultaneamente aos demais parâmetros da regressão. O modelo estatístico proposto por TOLER (1990) para descrever a adaptabilidade e estabilidade fenotípica é:

$$
\begin{aligned}
& Y_{i j}=\alpha_{i}+\left[Z_{j} \beta_{1 i}+\left(1-Z_{j}\right) \beta_{2 i}\right] \mu_{j}+\varepsilon_{i j} \text {, em que: } \\
& Y_{i j} \text { : é a resposta média do genótipo i no }
\end{aligned}
$$
ambiente $\mathrm{j}$ ( $\mathrm{i}=1,2, \ldots, \mathrm{p}$ genótipos; $\mathrm{e} \mathrm{j}=1,2, \ldots, \mathrm{q}$ ambientes);

$\alpha_{\mathrm{i}}$ : é o parâmetro que reflete o valor da resposta do genótipo i no ambiente de produtividade média $\left(\mu_{\mathrm{j}}=0\right)$;

$\beta_{1 \mathrm{i}}$ e $\beta_{2 \mathrm{i}}$ : são parâmetros que refletem a sensibilidade de resposta do genótipo i nos ambientes de baixa $\left(\mu_{\mathrm{j}} \leq 0\right)$ e de alta $\left(\mu_{\mathrm{j}}{ }^{3} 0\right)$ produtividade média respectivamente; ambiente $\mathrm{j}$;

$\mu_{\mathrm{j}}$ : é o parâmetro que reflete o efeito do

$\varepsilon_{\mathrm{ij}}$ : é o erro experimental médio;

$Z_{j}$ : é uma variável indicadora dummy, sendo $Z_{j}=1$ quando $\mu_{j} \leq 0$, e $Z_{j}=0$ quando $\mu_{j}>0$.

Para obtenção das estimativas de $\alpha_{i}, \beta_{1 i}, \beta_{2 i}$ e $\mu_{\mathrm{j}}$, foi utilizado o software Estabilidade, desenvolvido por Ferreira e Zambalde (1997), que utiliza a técnica de quadrados mínimos nãolineares, por meio de processos iterativos, empregando-se, para estimação desses parâmetros, o método de Gauss-Newton modificado (Rosse e VENCOVSKY, 2000; Rosse et al. 2002).

Os testes de hipóteses para classificação dos genótipos nos diferentes padrões de resposta adaptativa preconizados por TOLER (1990) basearamse na estatística $t$ de Student, tendo também sido implementado no mesmo programa. A primeira hipótese testada é $\mathrm{H}_{0}$ : $\beta_{1 \mathrm{i}}=\beta_{2 \mathrm{i}}$, então, se o teste for significativo, rejeita-se a hipótese, aceitando-se $\mathrm{H}_{\mathrm{a}}$ : $\beta_{1 \mathrm{i}}$ $\neq \beta_{2 \mathrm{i}}$, ocorrendo assim dois segmentos de reta, o que determina o modelo como bissegmentado. Nesse caso, o genótipo será classificado como grupo $\mathrm{A}$, se o padrão de resposta for $\beta_{1 \mathrm{i}}<1<\beta_{2 \mathrm{i}}$, obtendo-se uma resposta convexa e duplamente desejável; ou o genótipo será classificado como grupo $\mathrm{E}$, se $\beta_{1 \mathrm{i}}>1>$ $\beta_{2 \mathrm{i}}$, obtendo-se uma resposta côncava duplamente indesejável. Quando a hipótese $\mathrm{H}_{0}$ : $\beta_{1 \mathrm{i}}=\beta_{2 \mathrm{i}}$ for aceita admite-se um único segmento de reta para explicar o comportamento dos genótipos (obtendo-se um $\beta_{\mathrm{i}}$ comum). Os genótipos, nesse caso, serão classificados como grupo B, C ou D. No grupo B, quando $\beta_{\mathrm{i}}$ comum for significativo e $\beta_{i}>1$. Como $C$, quando $\beta_{i}=1$ e como $\mathrm{D}$ quando $\beta_{\mathrm{i}}$ comum for significativo e $\beta_{\mathrm{i}}<1$.

A interpretação da qualidade ambiental $\mathrm{m}_{\mathrm{j}}$ para esta análise é a mesma quando se interpreta $I_{j}$ de Eberhart e Russell (1966). Ressalva-se que nos modelos de regressão linear, a medida de qualidade ambiental (variável independente da regressão, Ij) é estimada em separado, antes da estimação dos coeficientes de regressão $(\beta)$. Já no procedimento de Toler (1990), essa medida denotada $\mu_{\mathrm{j}}$, é estimada simultaneamente aos demais parâmetros da regressão (ambiente favorável $\mu \mathrm{j}>0$; ambiente desfavorável $\mu \mathrm{j}<0)$; a diferença é que $\mu_{\mathrm{j}}(\mathrm{j}=1,2,3 \ldots \mathrm{q})$ são parâmetros estimados pelo modelo.

A equação $Y_{i j}=\alpha_{i}+\left[Z_{j} \beta_{1 i}+\left(1-Z_{j}\right) \beta_{2 i}\right] \mu_{j}$, pode ser reduzida a $Y_{i j}=\alpha_{i}+\beta_{i} \mu_{j}+\varepsilon_{i j}$, quando $\beta_{1 i}=\beta_{2 i}=\beta_{i}$. Para verificar se a primeira equação pode ser representada por uma única linha de regressão, devese comparar a igualdade entre $\beta_{1 \mathrm{i}}$ e $\beta_{2 \mathrm{i}}$. Para isso, é feito um teste de hipótese $\mathrm{H}_{0}\left(\beta_{1 \mathrm{i}}=\beta_{2 \mathrm{i}}\right)$ que permitirá determinar se o comportamento de um dado genótipo pode ser representado por uma reta de regressão ou não, ou seja, o modelo será uni ou bissegmentado. Através do modelo $Y_{i j}=\alpha_{i}+\left[Z_{j} \beta_{1 i}+\left(1-Z_{j}\right) \beta_{2 i}\right] \mu_{j}$, TOLER (1990) afirma ser possível classificar os genótipos com base nos padrões de resposta através dos testes de hipóteses, da seguinte forma:

\section{Grupo Critério}

A: $\quad$ Rejeita-se $H\left(\beta_{1}=\beta_{2}\right)$ e aceita-se $\beta_{1}<1<\beta_{2}$;

B: $\quad$ Não se rejeita $H\left(\beta_{1}=\beta_{2}\right)$ e rejeita-se $H(\beta=1)$, sendo o comum $\beta>1$;

C: $\quad$ Não se rejeita $H\left(\beta_{1}=\beta_{2}\right)$ e não se rejeita $H$ $(\beta=1)$;

D: $\quad$ Não se rejeita $H\left(\beta_{1}=\beta_{2}\right)$ e rejeita-se $H(\beta=1)$, sendo o comum $\beta<1$;

E: $\quad$ Rejeita-se $H\left(\beta_{1}=\beta_{2}\right)$ e aceita-se $\beta_{1}>1>\beta_{2}$.

TOLER (1990) descreve os significados práticos desses cinco grupos da seguinte forma: A é a resposta convexa e duplamente desejável; B é a resposta linear simples e desejável somente em ambientes de alta qualidade; $C$ é a resposta linear simples não desviando da resposta média; D é a resposta linear simples e desejável só em ambientes de baixa qualidade e E é a resposta côncava e duplamente indesejável.

Em cada análise de estabilidade, os materiais genéticos são enquadrados nos grupos de $\mathrm{A}$ a $\mathrm{E}$, conforme o padrão de resposta. 
Uma resposta convexa ou padrão de resposta duplamente desejável é, portanto, caracterizada quando o genótipo apresenta baixa responsividade nos ambientes desfavoráveis $(\mu j<0)$ e passa a responder satisfatoriamente quando essas condições tornam-se favoráveis $(\mu j>0)$. Uma resposta côncava ou padrão de resposta duplamente indesejável, segundo a classificação de TOLER (1990), caracteriza-se quando o genótipo é altamente responsivo aos ambientes de baixa qualidade e pouco responsivo em ambientes com qualidade acima dessas condições.

\section{RESULTADOS E DISCUSSÃO}

Os resultados observados nas análises de variância estão relacionados na tabela 1 . Os efeitos da interação de genótipo com ambientes (GxA), o efeito de ambientes e o efeito de genótipos foram significativos pelo teste F, para todos os ciclos de maturação. Esses resultados indicaram a existência de diferenças significativas, a 1\% de probabilidade, entre os genótipos avaliados e entre os ambientes, bem como efeitos significativos da interação GxA. Logo, houve influências diferenciadas dos ambientes, dificultando uma recomendação única de cultivares para toda a região em estudo. Neste caso, recomenda-se estabelecer um estudo detalhado do efeito da interação.

Verificou-se que os ambientes Costa Rica (MS601), Chapadão do Sul (MS-621) e Sorriso (MT-528) foram os que mais contribuíram para a diminuição da média ambiental, com estimativas de índices ambientais negativas $(\hat{\mu} j>0$, Tabela 2$)$, sendo Chapadão do Sul o mais desfavorável (maior valor negativo). Nos ambientes São Gabriel D'Oeste (MT641), Rondonópolis (MT-111), Pedra Preta (MT-221) e Campo Novo do Parecis (MT-701), contrariamente, as estimativas foram $\hat{\mu} j>0$, com destaque para o ambiente Pedra Preta, com a maior estimativa, o que contribuiu, para o aumento da média geral de ambientes. A amplitude da estimativa de qualidade ambiental $(\hat{\mu} \mathrm{j})$, entre os locais de maior e de menor produtividade, para o ciclo precoce foi de $733 \mathrm{~kg} \cdot \mathrm{ha}^{-1}$, para o ciclo médio $1162 \mathrm{~kg} \cdot \mathrm{ha}^{-1}$ e para o ciclo tardio $1.433 \mathrm{~kg} \mathrm{ha}^{-1}$.

Nas tabelas 3, 4 e 5, verifica-se o enquadramento dos genótipos de ciclo precoce, médio e tardio nos cinco grupos propostos pela metodologia respectivamente. Nos genótipos MSOY-8329, Milena, FMT-42313, Emgopa-315, FMT-41101, Garantia, Emgopa-313, FMT-42302 e Saíra verificou-se padrão de resposta convexo (grupo A), considerado duplamente desejável segundo Toler (1990). Nos genótipos FMT-41105, MSOY-8550, Crixás, Luziânia, FMT-42175, UFV-18, Xingu, Anhumas e FMT-43179 observou-se padrão de resposta côncavo (grupo E) duplamente indesejável segundo o método empregado.

Genótipos do grupo A são considerados aqueles que se adaptam bem em ambientes favoráveis, sendo responsivos em ambientes de alta qualidade. De acordo com a interpretação da metodologia, em genótipos do grupo A ocorrem comportamento que satisfaz ao conceito de genótipo ideal, em termos de adaptabilidade. Esses genótipos são exigentes em níveis de qualidade ambiental para expressarem todo seu potencial genético de produtividade. Rosse e VenCOVSKY (2000) esclarecem que, para alcançar esse potencial, é necessário o uso de altas tecnologias nos ambientes, pois, sob condições adversas com $\hat{\mu} \mathrm{j}<0$ (Costa Rica, Chapadão do Sul e Sorriso), tais genótipos revelam baixa responsividade. Genótipos assim não seriam os mais indicados para ambientes que utilizam poucos recursos tecnológicos. Genótipos do grupo E são considerados de resposta côncava e duplamente indesejável. Uma resposta côncava nesse método está relacionada ao genótipo com sensibilidade acima dessas condições médias, pouco responsivo à melhoria ambiental em condições favoráveis. Na amplitude de diferença entre os índices ambientais $(\mu \mathrm{j})$ constata-se a divergência e contrastes entre os locais de testes onde são realizados os ensaios; assim, quanto mais distintos, melhor será a discriminação dos genótipos em estudo quanto a sua adaptabilidade e estabilidade de comportamento para fins de recomendação.

Tabela 1. Resumo da análise conjunta de variância para produtividade de grãos $\left(\mathrm{kg} \mathrm{ha}^{-1}\right)$, em diferentes genótipos de soja, de três ciclos de maturação, na safra 2002/2003

\begin{tabular}{lcccccc}
\hline \multirow{2}{*}{ Fontes de Variação } & \multicolumn{5}{c}{ Quadrados Médios } \\
\cline { 2 - 7 } & GL & Ciclo Precoce & GL & Ciclo Médio & GL & Ciclo Tardio \\
\hline Ambiente (A) & 6 & $4461757,41^{* *}$ & 6 & $11688226,51^{* *}$ & 6 & $20488497,10^{* *}$ \\
Genótipo (G) & 14 & $658257,68^{* *}$ & 23 & $454011,44^{* *}$ & 29 & $276813,00^{* *}$ \\
Interação G x A & 70 & $268610,81^{* *}$ & 138 & $283538,02^{* *}$ & 174 & $186244,10^{* *}$ \\
Resíduo Médio & 158 & 164187,95 & 325 & 122939,10 & 365 & 125536,80 \\
$\left(\mathrm{~kg} \cdot\right.$ ha $\left.^{-1}\right)$ & & 2907,35 & & 2924,08 & 2837,46 \\
CV (\%) & & 13,94 & & 11,99 & 12,48 \\
\hline
\end{tabular}


Tabela 2. Resultados médios de produtividade de grãos $\left(\mathrm{kg} \mathrm{ha}^{-1}\right)$ em soja, por ciclo de maturação e ambiente, e índices de qualidade ambiental ( $\hat{\mu} \mathrm{j})$ estimados pelo método de Toler (1990), em ensaios de competição de cultivares, em Mato Grosso e Mato Grosso do Sul (2002/2003)

\begin{tabular}{|c|c|c|c|}
\hline Identificação & Ambiente & Média Genotípica & Índice de qualidade ambiental \\
\hline & & Ciclo Precoce & \\
\hline MS-601 & Costa Rica & 2641,09 & $-286,87$ \\
\hline MS-621 & Chapadão do Sul & 2420,11 & $-427,35$ \\
\hline MS-641 & São Gabriel D'Oeste & 3098,53 & 257,45 \\
\hline MT-111 & Rondonópolis & 3159,44 & 153,66 \\
\hline MT-221 & Pedra Preta & 3209,29 & 306,11 \\
\hline MT-528 & Sorriso & 2691,45 & $-254,08$ \\
\hline \multirow[t]{2}{*}{ MT-701 } & Campo Novo do Parecis & 3131,52 & 251,08 \\
\hline & & Ciclo Médio & \\
\hline MS-601 & Costa Rica & 2822,26 & $-131,39$ \\
\hline MS-621 & Chapadão do Sul & 2205,10 & $-744,68$ \\
\hline MS-641 & São Gabriel D'Oeste & 3129,23 & 140,86 \\
\hline MT-111 & Rondonópolis & 3198,58 & 380,11 \\
\hline MT-221 & Pedra Preta & 3428,67 & 417,73 \\
\hline MT-528 & Sorriso & 2622,23 & $-210,46$ \\
\hline \multirow[t]{2}{*}{ MT-701 } & Campo Novo do Parecis & 3062,47 & 147,83 \\
\hline & & Ciclo Tardio & \\
\hline MS-601 & Costa Rica & 2697,62 & $-163,61$ \\
\hline MS-621 & Chapadão do Sul & 2211,52 & $-622,35$ \\
\hline MS-641 & São Gabriel D'Oeste & 3137,50 & 311,23 \\
\hline MT-111 & Rondonópolis & 3618,50 & 810,57 \\
\hline MT-221 & Pedra Preta & 3081,58 & 206,46 \\
\hline MT-528 & Sorriso & 2328,81 & $-454,71$ \\
\hline MT-701 & Campo Novo do Parecis & 2786,66 & $-87,59$ \\
\hline
\end{tabular}

Tabela 3. Médias observadas $\left(\bar{Y}_{i}\right)$ e estimativas dos parâmetros do modelo não-linear de Toler $\left(\hat{\alpha}_{i}, \hat{\beta}_{1 \mathrm{i}}, \hat{\beta}_{2 \mathrm{i}}\right)$, para produtividade de grãos $\left(\mathrm{kg}_{\mathrm{g}} \mathrm{ha}^{-1}\right)$ de genótipos de soja de ciclo precoce, e seus respectivos padrões de resposta (grupo) segundo a metodologia (dados da safra 2002/2003, em Mato Grosso e Mato Grosso do Sul)

\begin{tabular}{|c|c|c|c|c|c|c|c|}
\hline N. ${ }^{\circ}$ de Identificação & Genótipo & $\overline{Y_{i}}$ & $\hat{\alpha}_{i}$ & $\hat{\beta}_{1 \mathrm{i}}$ & $\hat{\beta}_{2 \mathrm{i}}$ & $\hat{\beta}_{\text {i comum }}$ & Grupo \\
\hline 7 & MATRINCHÃ & 3177,97 & 3177,97 & 1,58 & $-0,45$ & 0,76 & $\mathrm{C}$ \\
\hline 11 & MSOY-8329 & 3119,38 & 2046,83 & $-1,80$ & $5,94^{*}$ & 1,45 & A \\
\hline 5 & CACHARA & 3074,77 & 3074,77 & 2,25 & $-0,33$ & 1,17 & $\mathrm{C}$ \\
\hline 8 & TUCUNARÉ & 3073,16 & 3073,16 & $-0,36$ & 1,52 & 0,40 & $\mathrm{D}$ \\
\hline 9 & DM-247 & 3046,94 & 3046,94 & 2,70 & $-1,87$ & 0,84 & $\mathrm{C}$ \\
\hline 6 & CONQUISTA & 2995,21 & 2995,21 & 1,05 & 1,66 & 1,30 & $\mathrm{C}$ \\
\hline 10 & MSOY-109 & 2937,01 & 2937,01 & 2,75 & $-1,31$ & 1,01 & $\mathrm{C}$ \\
\hline 4 & AV-2056/7 & 2903,92 & 2903,92 & 1,41 & 0,30 & 0,92 & $\mathrm{C}$ \\
\hline 3 & AV-1AE4B810 & 2902,90 & 2902,90 & 1,77 & $-1,07$ & 0,69 & $\mathrm{C}$ \\
\hline 15 & FMT-41102 & 2839,32 & 2839,32 & 2,16 & $-0,56$ & 1,04 & $\mathrm{C}$ \\
\hline 14 & FMT-41105 & 2802,58 & 3830,43 & $4,61^{*}$ & $-2,81$ & 1,56 & $\mathrm{E}$ \\
\hline 2 & AV-1043Rch & 2776,30 & 2776,30 & 0,77 & 1,31 & 1,02 & $\mathrm{C}$ \\
\hline 12 & UFV-17 & 2723,78 & 2723,78 & 0,84 & 0,99 & 0,89 & $\mathrm{C}$ \\
\hline 1 & MILENA & 2662,72 & 1101,35 & $-3,74$ & $7,53^{*}$ & 0,82 & A \\
\hline 13 & UFV-19 & 2574,18 & 2574,18 & $-1,03$ & 4,13 & 1,04 & $\mathrm{C}$ \\
\hline
\end{tabular}


Tabela 4. Médias observadas $\left(\bar{Y}_{i}\right)$ e estimativas dos parâmetros do modelo não-linear de Toler $\left(\hat{\alpha}_{i}, \hat{\beta}_{1 i}, \hat{\beta}_{2 i}\right)$,para produtividade de grãos ( $\mathrm{kg} \cdot \mathrm{ha}^{-1}$ ) de genótipos de soja de ciclo médio, e seus respectivos padrões de resposta (grupo) segundo a metodologia (dados da safra 2002/2003, em Mato Grosso e Mato Grosso do Sul)

\begin{tabular}{|c|c|c|c|c|c|c|c|}
\hline N. ${ }^{\circ}$ de Identificação & Genótipo & $\bar{Y}_{\mathrm{i}}$ & $\hat{\alpha}_{i}$ & $\hat{\beta}_{1 \mathrm{i}}$ & $\hat{\beta}_{2 \mathrm{i}}$ & $\hat{\beta}_{\text {i comum }}$ & Grupo \\
\hline 23 & FMT-42313 & 3178,84 & 2774,32 & $-0,34$ & $2,26^{* *}$ & 0,56 & A \\
\hline 22 & PINTADO & 3134,10 & 3134,10 & 0,45 & 1,09 & 0,63 & $\mathrm{C}$ \\
\hline 12 & MSOY-8411 & 3106,25 & 3106,25 & 1,55 & 2,02 & 1,71 & $\mathrm{~B}$ \\
\hline 24 & FMT-47101 & 3074,54 & 3074,54 & $-0,07$ & 0,98 & 0,32 & $\mathrm{D}$ \\
\hline 1 & EMGOPA-315 & 3047,19 & 2781,38 & 1,14 & $2,85^{* *}$ & 1,68 & $\mathrm{~A}$ \\
\hline 13 & MSOY-8550 & 3038,50 & 3455,03 & $2,06^{* *}$ & $-0,61$ & 1,08 & $\mathrm{E}$ \\
\hline 7 & CRIXÁS & 3025,77 & 3145,57 & $1,87^{*}$ & 1,09 & 1,56 & $\mathrm{E}$ \\
\hline 3 & GARANTIA & 3002,13 & 2539,22 & $-0,76$ & $2,21^{* *}$ & 0,35 & A \\
\hline 16 & FMT-42111 & 2980,13 & 2980,13 & 0,57 & 1,06 & 0,76 & $\mathrm{D}$ \\
\hline 18 & FMT-41101 & 2976,76 & 2722,13 & $-0,30$ & $1,33^{* *}$ & 0,20 & A \\
\hline 21 & FMT-42107 & 2975,07 & 2975,07 & 1,65 & 0,86 & 1,34 & $\mathrm{C}$ \\
\hline 9 & STB & 2973,28 & 2973,28 & 1,25 & 0,93 & 1,01 & $\mathrm{C}$ \\
\hline 10 & TAQUARI & 2956,02 & 2956,02 & 0,65 & 1,69 & 1,02 & $\mathrm{C}$ \\
\hline 11 & PIAPARA & 2922,32 & 2922,32 & 1,45 & 0,61 & 1,07 & $\mathrm{C}$ \\
\hline 4 & LUZIANIA & 2896,70 & 3171,12 & $1,33^{*}$ & $-0,43$ & 0,81 & $\mathrm{E}$ \\
\hline 19 & FMT-42307 & 2868,65 & 2868,65 & 1,55 & 0,28 & 1,19 & $\mathrm{C}$ \\
\hline 5 & CELESTE & 2864,08 & 2864,08 & 0,35 & 1,39 & 0,77 & $\mathrm{C}$ \\
\hline 15 & FMT-42175 & 2846,75 & 3030,86 & $1,86^{*}$ & 0,67 & 1,41 & $\mathrm{E}$ \\
\hline 14 & SUPREMA & 2830,20 & 2830,20 & 1,56 & 0,25 & 1,09 & $\mathrm{C}$ \\
\hline 20 & FMT-42108 & 2820,66 & 2820,66 & 0,67 & 0,56 & 0,67 & $\mathrm{C}$ \\
\hline 2 & UFV-18 & 2704,06 & 3138,62 & $1,94^{*}$ & $-0,85$ & 0,99 & $\mathrm{E}$ \\
\hline 17 & FMT-42109 & 2680,15 & 2680,15 & 0,39 & 1,84 & 0,90 & $\mathrm{C}$ \\
\hline 6 & GUAPORÉ & 2640,67 & 2640,67 & 1,02 & 1,76 & 1,35 & $\mathrm{C}$ \\
\hline 8 & XINGÚ & 2634,98 & 2952,63 & $2,10^{* *}$ & 0,06 & 1,34 & $\mathrm{E}$ \\
\hline
\end{tabular}

Os genótipos do grupo $C$ com valores $\hat{\beta}_{i}$ não diferem estatisticamente de 1,0 , ou são iguais à unidade. Seu desempenho acompanha aquele da média do ambiente. Segundo Rosse e Vencovsky (2000), parte de uma produtividade baixa atinge, no fim, níveis satisfatórios, além de bom ajuste do modelo ao conjunto de dados, o que significa alta previsibilidade. Genótipos com esse tipo de comportamento são aqueles com grau de mudança das características individuais em ambientes diferentes e plasticidade fenotípica, ou seja, maior estabilidade (BRADSHAW, 1965). Nesses ensaios, podese destacar a cultivar Conquista, aqui classificada como padrão C; de acordo com alguns autores (Prado et al. 2001; PACHECO et al. 2003; Morais et al 2004; PACHECo et al. 2005; Morais et al. 2005; Morais 2005) é de produtividade média e pouca variabilidade mesmo quando cultivada em ambientes distintos, ou seja, uma cultivar de alta estabilidade fenotípica.
O favorecimento à detecção de comportamento unissegmentado em maiores proporções aos genótipos de soja com padrão de resposta $\mathrm{C}$, sendo $73 \%$ de ciclo precoce, $46 \%$ de ciclo médio e $63 \%$ de ciclo tardio (Tabela 6), para os ensaios em estudo, pode ser atribuído à base genética estreita dos genótipos cultivados (НіRомото e Vello, 1996). Tal característica leva à explicação do comportamento médio geral e produtividades próximas desses genótipos, gerando assim dificuldade em se destacar genótipos de comportamento bissegmentado, devido à sua pouca variabilidade, neste caso é o que ocorre com a soja cultivada. Segundo TOLER (1990), em espécies melhoradas tende a ocorrer comportamento que pode ser mais bem explicado por um único segmento de reta, em virtude dos níveis de produtividade dos genótipos nos ambientes serem semelhantes e com padrões de resposta similares. Espécies com menor grau de melhoramento e picos de produtividades, bem diferentes de um ambiente para outro, podem resultar em dois segmentos de reta. 
Tabela 5. Médias observadas $\left(\bar{Y}_{i}\right)$ e estimativas dos parâmetros do modelo não-linear de Toler $\left(\hat{\alpha}_{i}, \hat{\beta}_{1 i}, \hat{\beta}_{2 i}\right)$, para produtividade de grãos (kg.ha-1) de genótipos de soja de ciclo tardio, e seus respectivos padrões de resposta (grupo) segundo a metodologia (dados da safra 2002/2003, em Mato Grosso e Mato Grosso do Sul)

\begin{tabular}{|c|c|c|c|c|c|c|c|}
\hline N. ${ }^{\circ}$ de Identificação & Genótipo & $\bar{Y}_{\mathrm{i}}$ & $\hat{\alpha}_{\mathrm{i}}$ & $\hat{\beta}_{1 \mathrm{i}}$ & $\hat{\beta}_{2 \mathrm{i}}$ & $\hat{\beta}_{\text {i comum }}$ & Grupo \\
\hline 23 & FMT-42152 & 3092,17 & 3092,17 & 1,46 & 0,78 & 1,08 & $\mathrm{C}$ \\
\hline 24 & FMT-43161 & 3054,13 & 3054,13 & 1,61 & 0,63 & 1,10 & $\mathrm{C}$ \\
\hline 28 & FMT-48101 & 3049,58 & 3049,58 & 0,76 & 0,53 & 0,65 & $\mathrm{D}$ \\
\hline 7 & MONARCA & 3032,04 & 3032,04 & 1,50 & 1,26 & 1,38 & $\mathrm{~B}$ \\
\hline 8 & MSOY-8800 & 3027,20 & 3027,20 & 0,83 & 1,00 & 0,92 & $\mathrm{C}$ \\
\hline 6 & JATAÍ & 2982,85 & 2982,85 & 0,67 & 1,18 & 0,95 & $\mathrm{C}$ \\
\hline 9 & MSOY-8866 & 2946,90 & 2946,90 & 1,08 & 0,87 & 0,96 & $\mathrm{C}$ \\
\hline 3 & DM-309 & 2941,31 & 2941,31 & 0,82 & 0,78 & 0,82 & $\mathrm{C}$ \\
\hline 15 & KAIABI & 2905,09 & 2905,09 & 1,75 & 1,13 & 1,40 & B \\
\hline 22 & FMT-42308 & 2878,43 & 2878,43 & 1,45 & 1,15 & 1,27 & $\mathrm{C}$ \\
\hline 16 & NAMBU & 2860,83 & 2860,83 & 1,21 & 0,98 & 1,08 & $\mathrm{C}$ \\
\hline 11 & TABARANA & 2855,82 & 2855,82 & 1,51 & 0,42 & 0,95 & $\mathrm{C}$ \\
\hline 5 & EMGOPA-313 & 2827,53 & 2424,37 & $-0,35$ & $1,77^{*}$ & 0,79 & $\mathrm{~A}$ \\
\hline 18 & MARITACA & 2822,19 & 2822,19 & 1,05 & 1,03 & 1,03 & $\mathrm{C}$ \\
\hline 4 & DM-339 & 2816,19 & 2816,19 & 1,21 & 0,69 & 0,92 & $\mathrm{C}$ \\
\hline 1 & DM-VITÓRIA & 2816,06 & 2816,06 & 0,38 & 1,57 & 1,02 & $\mathrm{C}$ \\
\hline 25 & FMT-42302 & 2808,11 & 2554,81 & 0,31 & $1,65^{*}$ & 1,03 & $\mathrm{~A}$ \\
\hline 13 & TUCANO & 2789,25 & 2789,25 & 2,29 & 0,74 & 1,47 & B \\
\hline 21 & ANHUMAS & 2768,63 & 3007,22 & $1,60^{*}$ & 0,34 & 0,92 & $\mathrm{E}$ \\
\hline 27 & FMT-42110 & 2765,97 & 2765,97 & 1,12 & 0,64 & 0,84 & $\mathrm{C}$ \\
\hline 19 & SABIA & 2751,62 & 2751,62 & 1,22 & 1,35 & 1,31 & $\mathrm{C}$ \\
\hline 2 & BARREIRAS & 2748,02 & 2748,02 & 0,11 & 0,89 & 0,52 & $\mathrm{D}$ \\
\hline 26 & FMT-43105 & 2736,20 & 2736,20 & 0,79 & 1,39 & 1,12 & $\mathrm{C}$ \\
\hline 30 & FMT-11220 & 2735,89 & 2735,89 & 0,42 & 0,44 & 0,43 & $\mathrm{D}$ \\
\hline 20 & SAIRA & 2735,00 & 2544,40 & 0,65 & $1,65^{*}$ & 1,20 & $\mathrm{~A}$ \\
\hline 17 & PERDIZ & 2697,68 & 2697,68 & 0,49 & 1,35 & 0,94 & $\mathrm{C}$ \\
\hline 14 & UIRAPURU & 2679,50 & 2679,50 & 1,34 & 0,68 & 0,99 & $\mathrm{C}$ \\
\hline 12 & MUTUM & 2674,95 & 2674,95 & 0,40 & 1,03 & 0,73 & $\mathrm{C}$ \\
\hline 29 & FMT-43179 & 2662,43 & 2917,18 & $1,66^{*}$ & 0,31 & 0,94 & $\mathrm{E}$ \\
\hline 10 & MSOY-8914 & 2661,97 & 2661,97 & 0,51 & 1,61 & 1,09 & $\mathrm{C}$ \\
\hline
\end{tabular}

As médias genotípicas estimadas pelo modelo de regressão $\left(\hat{\alpha}_{i}\right)$, para genótipos classificados com padrão $\mathrm{A}$ e $\mathrm{E}$, não correspondem às médias genotípicas originais $\left(\bar{Y}_{\mathrm{i} .}\right)$ (Tabelas 3, 4 e 5). Nas linhagens FMT-42313 e FMT-41101 de padrão A ocorreram perdas de produtividade, respectivamente, de $12 \%, 72 \%$ e $8,5 \%$, Nesse mesmo grupo, nas cultivares Emgopa-315 e Garantia houve perdas de $8,7 \%$ e $15,4 \%$. Nos genótipos de padrão $\mathrm{E}$ os ganhos foram de 13,7\% para MSOY-8550, 3,9\% para Crixás, 9,5\% para Luziânia, 16,1\% UFV-18, 12,1\% Xingú e FMT-42175 (15) com ganho de 6,5\%.

Nos grupos de genótipos de diferentes ciclos, observou-se, com base no método de regressão nãolinear proposto por TOLER (1990), que na maioria deles ocorreu comportamento que pode ser explicado por apenas um segmento de reta. Além disso, em sua maioria, os genótipos com valores de $\beta_{\mathrm{i}}$ comum iguais à unidade acompanharam o desempenho médio dos ambientes. A regressão de um só segmento, de fato, decorre de genótipos com padrões de resposta similares. Aliado a isso, nessas circunstâncias, espera-se a maior parte deles com $\beta$ comum $=1,0$. Esses resultados contrariam aqueles observados por RosSE e VENCOVSKY (2000), na cultura do feijão, em que apenas 35\% dos genótipos estudados enquadram-se como unissegmentados; corroboram, também, com os resultados descritos por Ribeiro et al. (2000), em milho, que observaram padrão de resposta unissegmentado em $90 \%$ dos genótipos estudados. Rosse et al. (2002) também constataram esse comportamento em $80 \%$ dos genótipos de cana-de açúcar, classificados como unissegmentados. 
Tabela 6. Número de genótipos de soja por ciclo de maturação, classificados de acordo com os níveis de produtividade e padrões de resposta, segundo a metodologia de Toler (1990)

\begin{tabular}{|c|c|c|c|c|c|c|c|}
\hline \multirow{2}{*}{ Ciclo } & \multicolumn{5}{|c|}{ Classes de produtividade $\left({ }^{1}\right)$} & \multirow{2}{*}{$\bar{x}$} & \multirow{2}{*}{$\mathrm{s}$} \\
\hline & A & B & $\mathrm{C}$ & $\mathrm{D}$ & $\mathrm{E}$ & & \\
\hline Precoce & & & & & & 2907,35 & 405,2011 \\
\hline Alta produtividade & 1 & 0 & 1 & 0 & 0 & & \\
\hline Média produtividade & 0 & 0 & 9 & 1 & 1 & & \\
\hline Alta produtividade & 1 & 0 & 1 & 0 & 0 & & \\
\hline Total & 2 & 0 & 11 & 1 & 1 & & \\
\hline \% dos genótipos & 13,3 & 0 & 73,3 & 6,6 & 6,6 & & \\
\hline Médio & & & & & & 2924,39 & 350,6267 \\
\hline Alta produtividade & 1 & 1 & 1 & 0 & 0 & & \\
\hline Média produtividade & 3 & 0 & 8 & 2 & 4 & & \\
\hline Alta produtividade & 0 & 0 & 2 & 0 & 2 & & \\
\hline Total & 4 & 1 & 11 & 2 & 6 & & \\
\hline$\%$ dos genótipos & 16,6 & 4,1 & 45,8 & 8,3 & 25 & & \\
\hline Tardio & & & & & & 2837,46 & 354,3117 \\
\hline Alta produtividade & 0 & 1 & 3 & 1 & 0 & & \\
\hline Média produtividade & 3 & 2 & 16 & 2 & 2 & & \\
\hline Alta produtividade & 0 & 0 & 0 & 0 & 0 & & \\
\hline Total & 3 & 3 & 19 & 3 & 2 & & \\
\hline$\%$ dos genótipos & 10,0 & 10,0 & 63,3 & 10,0 & 6,6 & & \\
\hline
\end{tabular}

Com detecção de $65 \%$ de genótipos de feijão classificados como bissegmentados, Rosse e VENCOVSKY (2000), em seu estudo, esclarecem essa ocorrência devido à espécie estudadada ser de ciclo anual e com desenvolvimento precoce, e mais sensível às oscilações ambientais. Assim, em sua produtividade ocorrem mudanças mais acentuadas quando o ambiente é alterado, o que pode ter, em parte, favorecido a ocorrência de muitos genótipos nos grupos bissegmentados. Os autores também refletem quanto ao caso de resultados ocorridos como nos trabalhos realizados por TOLER (1990) e RibeIro et al. (2000), nos quais a maioria dos genótipos foi enquadrada nos grupos B, C e D. Esses trabalhos envolveram espécies já em elevado grau de melhoramento, e que mantêm comportamento fisiológico semelhante, impedindo de certa forma atingirem um platô máximo de produtividade.

Quanto à interpretação bissegmentada dos coeficientes e do modelo, praticamente é a mesma verificada por Verma et al. (1978), Silva e BarReto (1985) e CRUZ et al. (1989). O coeficiente identifica o padrão de resposta dos genótipos nos ambientes abaixo da média geral de todos os ambientes $\left(\mu_{\mathrm{j}} \leq 0\right)$, ou seja, condições ambientais de baixa tecnologia. Para o coeficiente, o padrão de resposta dos genótipos está acima da média geral de todos os ambientes $\left(\mu_{\mathrm{j}} \geq 0\right)$.
Genótipos de soja de padrão A corresponderam a $13 \%$ do total do ciclo precoce, $17 \%$ do total de ciclo médio e $10 \%$ de genótipos de ciclo tardio (Tabela 6). Esses genótipos são de menor $\beta_{1}$. que a unidade e $\beta_{2}$. maior, indicando que, em seu comportamento produtivo, suportam bem as condições adversas dos ambientes; quando essas condições tendem à melhoria, esses genótipos respondem satisfatoriamente; acarretando, assim, incrementos em suas produtividades nos locais de qualidade superior. No total de genótipos descritos neste trabalho, nove são descritos como de padrão A de resposta, com destaque para a linhagem FMT42313 de ciclo precoce que atingiu alta produtividade seguida dos genótipos MSOY-8329 (precoce), Emgopa315, Garantia e FMT-41101 de ciclo médio (Tabelas 3 e 4).

Quanto ao padrão $E$, há destaque para as cultivares MSOY-8550 e Crixás de ciclo precoce em relação às maiores produtividades. Genótipos enquadrados no grupo E, que se caracterizam por serem bastante responsivos aos ambientes que empregam baixa tecnologia e passam a ser menos responsivos em produtividade, quando começam a explorar os ambientes superiores, mais tecnificados, revelam algumas vantagens em relação aos do grupo A. Alguns genótipos de padrão E não atingem o 
"topo" de produtividade, mas patamares mais elevados e satisfatórios de produtividade média como é o caso de MSOY-8550 e Crixás. Segundo Rosse (1999), nessa situação, a definição de que genótipos de comportamento ideal seriam preferencialmente aqueles enquadrados no grupo A, não seria muito apropriada. Deste modo, os materiais genéticos de padrão E seriam, então, mais aconselháveis para os pequenos agricultores, com baixa tecnologia onde não necessariamente seriam buscadas condições ótimas.

Na figura 1, a linhagem FMT-42302 ilustra o comportamento de um genótipo de padrão E, partindo dos ambientes inferiores com um nível baixo de produtividade, chegando-se a produtividades acima da média genotípica. Ao contrário desse genótipo, com a cultivar Anhumas de padrão A, nos ambientes desfavoráveis ocorreram maiores produtividades. Quando há melhorias dessas condições, essa cultivar atinge no $\mathrm{fm}$ a maior de todas as produtividades chegando a aproximar-se de $4.000 \mathrm{~kg} \cdot \mathrm{ha}^{-1}$.

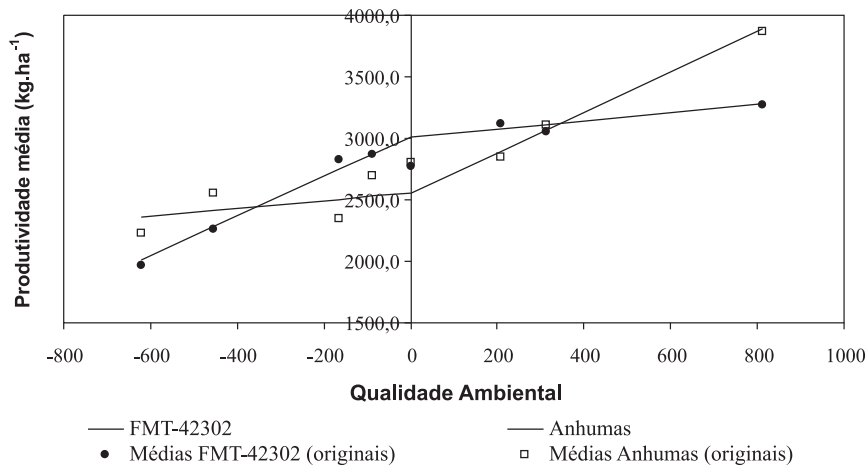

Figura 1. Comportamento de genótipos de soja do ciclo tardio enquadrados nos grupos bisegmentados, padrão A (Anhumas) e padrão E (FMT-42302), avaliados na safra 2002/03, em sete ambientes dos Estados de Mato Grosso e Mato Grosso do Sul, Brasil.

Segundo DuARTE (1988), em modelos lineares de estabilidade observa-se alta associação positiva entre a média dos genótipos e seu coeficiente de regressão. Neste estudo, genótipos de padrão $B\left(\beta_{i}>1\right)$ estão sempre associados a médias altas, acima do grupo $\mathrm{D}\left(\beta_{\mathrm{i}}<1\right)$, indicando médias mais baixas na maioria dos casos. Assim, podem-se constatar esses resultados para alguns genótipos nos gráficos da figura 2.

Nesses gráficos, observa-se o comportamento de genótipos enquadrados nos padrões B, C e D, em seus extremos de produtividade. Os genótipos de padrão B caracterizam-se por pela alta responsividade aos ambientes. É um tipo de genótipo mais exigente, alcançando boa produtividade nos ambientes mais tecnificados. Observa-se pelos gráficos que esses genótipos possuem produtividades muito inferior em locais de baixos índices de qualidade ambiental, mas, quando cultivados em condições melhores chegam a atingir $100 \%$ a mais em produtividade média. Esses genótipos têm em comum a capacidade de explorarem de modo eficiente os ambientes favoráveis $\left(\beta_{\mathrm{i}}>1\right)$. Os genótipos de padrão $\mathrm{D}$, como constatado nos gráficos, indicam especificidade para aqueles ambientes que empregam baixos níveis tecnológicos (baixo índice de qualidade ambiental), onde conseguem ter produtividades médias mais expressivas que genótipos de padrão B.
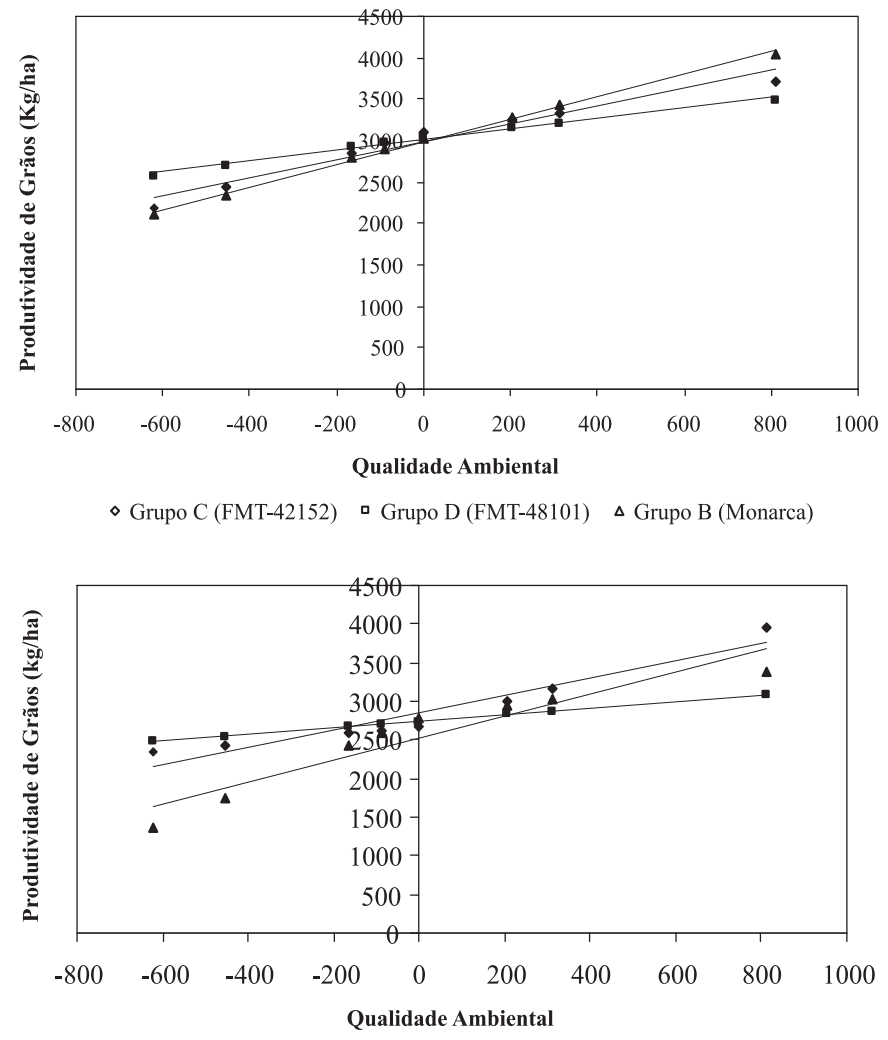

- Grupo C (MSOY-8914) $\square$ Grupo D (FMT-11220) $\Delta$ Grupo B (Tucano)

Figura 2. Comportamento de genótipos de soja do ciclo tardio, enquadrados nos grupos unissegmentados (padrão B, C e D), avaliados na safra 2002/2003, em sete ambientes dos Estados de Mato Grosso e Mato Grosso do Sul, Brasil.

\section{CONCLUSÃO}

Considera-se que o método de TOLER (1990) tem a capacidade de caracterizar melhor o comportamento dos genótipos e detectar linhagens promissoras dentro de cada padrão de resposta. De acordo com a necessidade do programa, o melhorista poderá selecionar, de fato, genótipos com potencial genético para serem usados em cruzamentos futuros com base nos padrões de resposta. 


\section{AGRADECIMENTOS}

Os autores agradecem ao Dr. Cláudio Takeda (Fundação de Apoio à Pesquisa Agropecuária do estado do Mato Grosso), que gentilmente cedeu os dados para esse estudo e ao Dr. João Batista Duarte (Escola de Agronomia e Engenharia de Alimentos - UFG) pelas sugestões e críticas.

\section{REFERÊNCIAS}

BRADSHAW, A. D. Evolutionary significance of phenotypic plasticity. Advances in Genetics, New York, v.13, p.115-153, 1965.

COCHRAN, W. G. The combination of estimates from different experiments. Biometrics, Arlington, v.10, p.101-129, 1954.

CRUZ, C. D.; TORRES, R. A. A. VENCOVSKY, R. An alternative approch to stability analysis proposed by Silva and Barreto. Revista Brasileira de Genética, Ribeirão Preto, v.12, p.567-580, 1989.

DUARTE, J. B. Estudo da adaptabilidade e estabilidade fenotípica em linhagens e cultivares de feijão mulatinho (Phaseolus vulgaris L.). 1988. 155f. Dissertação (Mestrado) Escola de Agronomia - UFG, Goiânia.

DUARTE, J. B.; VENCOVSKY, R. Interação genótipos $\mathbf{x}$ ambientes: uma introdução à análise "AMMI". Ribeirão Preto: Sociedade Brasileira de Genética, 1999. 60p. (Monografias, 9)

EBERHART, S. A.; RUSSELL, W. A. Stability parametres for comparing varieties. Crop Science, Madison, v.6, n.1, p.36-40. 1966.

FERREIRA, D. F.;ZAMBALDE, A.. L. Simplificação das análises de algumas técnicas especiais da experimentação agropecuária no Mapgen e softwares correlatos. In: CONGRESSO DA SOCIEDADE BRASILEIRA DE INFORMÁTICA APLICADA À AGROPECUÁRIA E À AGROINDÚSTRIA, 1., Belo Horizonte, 1997. Anais... Belo Horizonte: SBI, 1997. p.285-291.

FINLAY, K. W.; WILKINSON, G. N. The analysis of adaptation in a plant breeding programme. Australian Journal of Agricultural Research, Collingwood, v.14, p.742-754. 1963.

HIROMOTO, D.M.; VELLO, N.A. Genetic base of Brazilian soybean cultivars. Revista Brasileira de Genética, Ribeirão Preto, v.9, n.2, p.295-306, 1986.

MORAIS, L. K. Adaptabilidade e estabilidade fenotípica em soja nos Estados de Mato Grosso e Mato Grosso do Sul. 2005. $115 \mathrm{f}$. Tese (Doutorado em Agronomia) - Escola de Agronomia e Engenharia de Alimentos - UFG, Goiânia.

MORAIS, L. K.; MOURA, M. F.; VENCOVSKY, R.; DUARTE, J. B.; TAKEDA, C.; PINHEIRO, J. B. Adaptabilidade e estabilidade fenotípica em soja utilizando regressão nãolinear. In: CONGRESSO BRASILEIRO DE GENÉTICA, 50., 2004, Florianópolis, SC. Resumos... Ribeirão Preto, SP: Sociedade Brasileira de Genética, 2004. p. 1302-1302.

MORAIS, L. K.; PINHEIRO, J. B. ; VENCOVSKY, R.; MOURA, M. F.; CHIORATO, A. F. Adaptabilidade e estabilidade fenotípica em soja para os estados de Mato Grosso e Mato Grosso do Sul. In: CONGRESSO BRASILEIRO DE MELHORAMENTO
DE PLANTAS, 3, 2005, Gramado, RS. Anais... Viçosa, MG: Sociedade Brasileira de Melhoramento de Plantas, 2005. CD.

PACHECO, R. M.; DUARTE, J. B.; ASSUNÇÃO, M. S.; NUNES JUNIOR, J.; CHAVES, A. A. P. Zoneamento e adaptação produtiva de genótipos de soja de ciclo médio de maturação para Goiás. Pesquisa Agropecuária Brasileira, Brasília, 2003, vol. 33, n. 1, p. 23-27.

PACHECO, R. M.; DUARTE, J. B.; VENCOVSKY, R.; PINHEIRO; J. B.; OLIVEIRA, A. B. Use of supplementary genotypes in AMMI analysis. Theoretical Applied Genetics, Stuttgart, 2005, v. 110, p. 812-818.

PLAISTED, R. L.; PETERSON, L. C. A technique for evaluating the ability of selection to yield consistently over locations. American Potato Journal, Minnesota, v.37, p.166-172. 1959.

PRADO, E. E., HIROMOTO, D. M., GODINHO, V. de P. C. Adaptabilidade e estabilidade de cultivares de soja em cinco épocas de plantio no cerrado de Rondônia. Pesquisa Agropecuária Brasileira, Brasília, 2001, vol. 36, no. 4, p. 625-635.

RIBEIRO, P. H. E.; RAMALHO, M. A. P.; FERREIRA, D. F. Adaptabilidade e estabilidade de genótipos de milho em diferentes condições ambientais. Pesquisa Agropecuária Brasileira, Brasília, v. 35, n. 11, 2000.

ROSSE, L. N. Modelo de regressão não-linear aplicado na avaliação da estabilidade fenotípica em plantas. 1999. 179 f., Tese (Doutorado). Escola Superior de Agricultura "Luiz de Queiroz" -USP, Piracicaba.

ROSSE, L. N.; VENCOVSKY, R. Modelo de regressão nãolinear aplicado ao estudo da estabilidade fenotípica de genótipos de feijão no Estado de São Paulo. Bragantia, Campinas, v.59, n.1, p.99-107. 2000.

ROSSE, L. N.; VENCOVSKY, R.; FERREIRA, D. F. Comparação de métodos de regressão para avaliar a estabilidade fenotípica em cana-de-açúcar. Pesquisa Agropecuária Brasileira, Brasília, v.37, n.1, p. 25-32. 2002.

SAS Institute. Software and services: system for Windows, versão 8.0: software. Cary, 2002.

SILVA, J. C.; BARRETO, J. N. Aplicação da regressão linear segmentada em estudos de interação genótipo por ambiente. In: SIMPÓSIO DE ESTATÍSTICA APLICADA À EXPERIMENTAÇÃO AGRONÔMICA, 1., Piracicaba, 1985. Anais... Campinas: Fundação Cargill, 1985. p.49-50.

TOLER, J. E. Patterns of genotypic performance over environmental arrays. 1990. 154p. Thesis (Ph.D)-Clemson University, Clemson.

VERMA, M. M.; CHAHAL, G. C.; MURTY, B. R. Limitation of conventional regression analysis: a proposed modification. Theoretical Applied Genetics, Stuttgart, v.53, n.2, p.89-91, 1978.

WRICKE, G. Zur Berechnung der Okovalens bei Sommerweizen und Hafer. Z. Pflanzenzuchtung, Berlin, v.52, p.127-138. 1964.

YATES, F.; COCHRAN, W. G. The analysis of groups of experiments. The Journal of Agricultural Science, Cambridge, v.28, n.4, p.556-580, 1938. 